\title{
Gestão das intervenções de prevenção e controle da resistência a antimicrobianos em hospitais: revisão de evidências
}

\author{
Roberta Crevelário de Melo, ${ }^{1}$ Bruna Carolina de Araújo, ${ }^{1}$ Maritsa Carla de Bortoli ${ }^{1}$ e Tereza \\ Setsuko Toma ${ }^{1}$
}

Como citar Melo RC, Araújo BC, Bortoli MC, Toma TS. Gestão das intervenções de prevenção e controle da resistência a antimicrobianos em hospitais: revisão de evidências. Rev Panam Salud Publica. 2020;44:e35. https://doi.org/10.26633/RPSP.2020.35

RESUMO

Objetivo. Identificar intervenções efetivas para enfrentar a resistência a antimicrobianos em hospitais e possíveis barreiras para sua implementação.

Métodos. Realizou-se uma síntese de evidências para a elaboração de políticas com base na metodologia proposta nas ferramentas SUPPORT. As buscas na literatura foram realizadas em novembro e dezembro de 2018, em 14 bases de dados. Um diálogo deliberativo, para identificar barreiras na implementação das intervenções, foi realizado presencialmente com 23 participantes (gestores, pesquisadores e profissionais de saúde) e 14 ouvintes, divididos em três grupos. A moderação foi feita por pesquisadores com experiência na condução de diálogos deliberativos.

Resultados. Foram identificadas 27 revisões sistemáticas sobre intervenções para gestão de antimicrobianos (programas de stewardship) combinadas e individuais. Essas intervenções abordaram estratégias de educação, sistemas eletrônicos, biomarcadores e diversas formas de manejo de antimicrobianos. As principais barreiras para a implementação das intervenções, identificadas por meio da literatura e do diálogo deliberativo, foram: falta de infraestrutura e recursos humanos, insatisfação do paciente em relação à conduta terapêutica; diferenças culturais da equipe multidisciplinar; sobrecarga de trabalho da equipe; falta de financiamento/planejamento.

Conclusão. A maioria das estratégias identificadas mostrou-se efetiva para gestão da resistência aos antimicrobianos em âmbito hospitalar. Deve-se enfatizar que a confiança nos resultados pode ser melhorada com novas pesquisas de maior qualidade metodológica.

Palavras-chave Resistência microbiana a medicamentos; gestão de antimicrobianos; hospitais; política informada por evidências.

A resistência aos antimicrobianos é um fenômeno que põe em risco a segurança da saúde das populações em todo o mundo (1-4). Há vários mecanismos pelos quais a resistência aos antimicrobianos pode ocorrer, incluindo a proliferação de bactérias resistentes no paciente e a seleção de cepas resistentes no indivíduo tratado, com disseminação para outras pessoas e lugares. A resistência aos antimicrobianos também pode ser desenvolvida naturalmente ao longo do tempo, pela exposição dos microrganismos a substâncias antimicrobianas encontradas em alimentos, pessoas, animais, plantas e ambiente. Esse processo faz com que os tratamentos se tornem prolongados, dificultando a adesão do paciente e contribuindo para o desenvolvimento e disseminação de infecções persistentes. Na área da saúde, os fatores que contribuem para a resistência aos antimicrobianos incluem o

\footnotetext{
Secretaria de Estado de Saúde, Instituto de Saúde, Centro de Tecnologias de Saúde para o SUS, São Paulo (SP), Brasil. $₫$ Bruna Carolina de Araújo, brucarujo@gmail.com
} 
uso inadequado de antibióticos, a internação hospitalar, sobretudo em unidades de terapia intensiva (UTI), o uso de acessórios invasivos de diagnóstico e tratamento, cirurgias, doenças intercorrentes, queimaduras e idade do paciente, entre outros $(5,6)$.

No mundo, os antimicrobianos são a segunda classe de medicamentos mais utilizada em hospitais, respondendo por $20 \%$ a $50 \%$ das despesas com medicamentos nesses serviços, além de serem também prescritos em larga escala em nível ambulatorial (7). Na América Latina, os estudos, apesar de escassos, indicam uma tendência de aumento na utilização de todas as classes de antimicrobianos no período de 1997 a 2007. Estudos observacionais estimaram que, em serviços de saúde na região, os antibióticos sejam utilizados de forma inadequada em $50 \%$ dos casos; e que a taxa de automedicação por parte dos pacientes varie de $20 \%$ a $40 \%$ (8).

A necessidade de enfrentar esse problema levou ao desenvolvimento de algumas estratégias, como, por exemplo, programas e intervenções para gestão do uso de antibióticos - os assim chamados programas de antimicrobial stewardship. Tais estratégias têm orientado o estabelecimento de iniciativas como as redes de vigilância da resistência aos antimicrobianos na Europa, Ásia Central e Europa Oriental, e América Latina $(4,9)$.

O termo antimicrobial stewardship tem sido aplicado em diferentes contextos de saúde como um conjunto de ações para promover o uso racional de antimicrobianos. Segundo alguns autores, os programas de stewardship têm o propósito de diminuir o consumo excessivo de antibióticos nos serviços de saúde e combater a resistência antimicrobiana de modo que a seleção, a dose, a via de administração e a duração da terapia sejam a base da terapêutica adequada (10-12) para se alcançar a máxima cura clínica ou a prevenção de infecção, limitar consequências não intencionais e garantir o uso de intervenções custo-efetivas (1315). Dyar et al. (16) definem os programas de stewardship como um "conjunto coerente de ações que promovem o uso de antimicrobianos de maneira a garantir o acesso sustentável a tratamento eficaz para todos os que precisam desses medicamentos" (p. 796).

$\mathrm{Na}$ América Latina, existem programas de stewardship, ou gestão, do uso de antimicrobianos em $46 \%$ dos hospitais, em comparação a 66\% na Europa e 67\% na América do Norte (8). No Brasil, com a finalidade de estabelecer ações de prevenção e controle da resistência microbiana nos serviços de saúde, a Agência Nacional de Vigilância Sanitária (ANVISA) elaborou em 2018 o Plano de Ação da Vigilância Sanitária em Resistência aos Antimicrobianos (17), o Plano Nacional para a Prevenção e o Controle da Resistência Microbiana nos Serviços de Saúde e a Rede de Resistência Microbiana (18). Entretanto, são escassos os estudos sobre programas de stewardship em serviços pediátricos, em departamentos de emergência e em países de baixa e média renda (19).

Este estudo foi realizado como resposta a uma demanda do Ministério da Saúde brasileiro por intervenções efetivas para enfrentar o problema da resistência aos antimicrobianos em serviços de saúde. Assim, o objetivo deste artigo é apresentar as intervenções de stewardship identificadas em revisões sistemáticas para enfrentamento da resistência microbiana em hospitais e barreiras para sua implementação.

\section{MATERIAIS E MÉTODOS}

Realizou-se uma síntese de evidências para a elaboração de políticas, conforme a metodologia proposta pelas ferramentas
SUPporting POlicy relevant Reviews and Trials (SUPPORT). Essa ferramenta pressupõe um ciclo no processo de tradução do conhecimento, com início na definição clara de um problema prioritário de saúde, seguida pelo desenvolvimento de uma síntese de evidências para políticas e pela condução de um diálogo deliberativo com representantes das partes interessadas no problema (20). A síntese de evidências para políticas (policy brief) é um documento preparado para atender a uma demanda da gestão do sistema de saúde no qual se oferecem opções de políticas identificadas a partir de revisões sistemáticas. Sua finalidade é informar sobre a urgência de um problema de saúde e a necessidade de adoção de opções, ou estratégias de intervenção, selecionadas por meio de processos sistemáticos e transparentes (21).

As buscas por evidências de pesquisa sobre opções para políticas foram realizadas em novembro e dezembro de 2018, nas seguintes bases de dados: Portal Regional da BVS (https:// bvsalud.org/), MEDLINE via PubMed (https: / www.ncbi.nlm. nih.gov/pubmed/), Health Systems Evidence (https://www. healthsystemsevidence.org/), PDQ Evidence (https://www. pdq-evidence.org), Center for Reviews and Dissemination (https://www.crd.york.ac.uk/CRDWeb/), Embase (https:// www.embase.com/login), Scopus (https://www.scopus. com/), Web of Science (http://www.webofscience.com/), Cochrane Collaboration (https://www.cochranelibrary.com/), Proquest Dissertations and Theses Global (https://www.proquest.com/products-services/pqdtglobal.html), SisREBRATS (http:/ / rebrats.saude.gov.br/sisrebrats), Biblioteca Digital Brasileira de Teses e Dissertações (http://bdtd.ibict.br/vufind/) e Joanna Briggs Institute (https://joannabriggs.org/ebp\#database). Os termos utilizados nas buscas foram "drug resistance", "antimicrobial resistance", "antibiotic resistance", "antimicrobial stewardship" (termo MeSH e entry terms "Stewardship, Antimicrobial", "Antibiotic Stewardship" e "Stewardship, Antibiotic"), "drug resistance, microbial", "'resistência aos antimicrobianos", "resistência microbiana", "resistência microbiana a medicamentos", "farmacorresistencia microbiana", "resistência a antibióticos", "resistência microbiana a antibióticos" e "resistência microbiana a drogas". As buscas se limitaram a revisões sistemáticas publicadas em inglês, espanhol e português. O processo de seleção foi realizado em duplicidade e de forma independente por duas das autoras (BCA e RCM), e as divergências foram sanadas por consenso. A qualidade metodológica das revisões sistemáticas foi classificada como alta (escore 8-11/11), moderada (5-7/11) ou baixa (0-4/11) a partir da ferramenta AMSTAR - Assessment of Multiple Systematic Reviews (22). Essa avaliação foi feita de forma independente por BCA, RCM, MCB e TST, sendo as divergências resolvidas por consenso. Os dados das revisões sistemáticas selecionadas foram extraídos em duplicidade e de forma independente por BCA e RCM, por meio de uma planilha contendo os seguintes itens: estudo (autor, ano); objetivo do estudo; características dos estudos; principais achados; considerações sobre implementação e equidade; lacunas de conhecimento; estudos realizados em países de baixa e média renda; último ano da busca.

O processo de identificação de barreiras e facilitadores para a implementação das estratégias foi realizado por meio de dados de literatura e de contribuições das partes interessadas (pesquisadores, profissionais e gestores de saúde), que participaram de um diálogo deliberativo para validação dos resultados da síntese de evidências para políticas. Os diálogos 
deliberativos permitem que as evidências de pesquisa sejam consideradas juntamente com as visões e experiências e o conhecimento implícito daqueles que estarão envolvidos com ou serão afetados pelas decisões futuras relacionadas a uma questão altamente prioritária. Os diálogos não têm um formato único, constituindo-se, normalmente, de uma reunião com atores-chave, que vão se expressar em relação a um documento previamente compartilhado sobre um tema específico. As reuniões são conduzidas por facilitadores e buscam um campo comum de troca e interação entre os atores-chave. A escolha dos participantes é fundamental para que se estabeleça um equilíbrio e para que todos possam se expressar (23). O diálogo deliberativo teve a participação de 23 pessoas interessadas no problema, entre gestores, pesquisadores e profissionais de saúde, e 14 ouvintes, divididos em três grupos. Cada grupo contou com a moderação de um pesquisador com experiência na condução de diálogos deliberativos e, posteriormente, houve uma plenária para socialização da discussão dos grupos. As contribuições dos participantes do diálogo deliberativo foram agrupadas em categorias e complementadas com informações da literatura sobre barreiras e facilitadores para a implementação das intervenções.

\section{RESULTADOS}

A revisão de literatura gerou 5462 artigos. Após o processo de seleção, 47 revisões sistemáticas foram analisadas e resultaram na elaboração de quatro opções para políticas: 1) programas de gestão com múltiplas intervenções combinadas para o uso racional de antimicrobianos em hospitais; 2) programas de gestão com uma intervenção individual para o uso racional de antimicrobianos em hospitais; 3) outros programas e intervenções (não de stewardship) para lidar com a resistência aos antimicrobianos em hospitais; 4) programa de gestão e intervenções para o uso racional de antimicrobianos na atenção primária, em ambulatórios, serviços de emergência e casas de repouso. $\mathrm{O}$ presente artigo se refere às opções 1 e 2 , ou seja, programas de gestão para o uso racional de antimicrobianos em hospitais desenvolvidos a partir da combinação de múltiplas intervenções ou de uma intervenção individual.

Para o foco deste artigo, foram selecionadas 27 revisões sistemáticas sobre programas ou intervenções denominados antimicrobial stewardship e implementados em ambiente hospitalar $(10-15,19,24-42)$. O fluxograma do processo de seleção é apresentado na figura 1 . A tabela 1 descreve as características

FIGURA 1. Fluxograma PRISMA adaptado

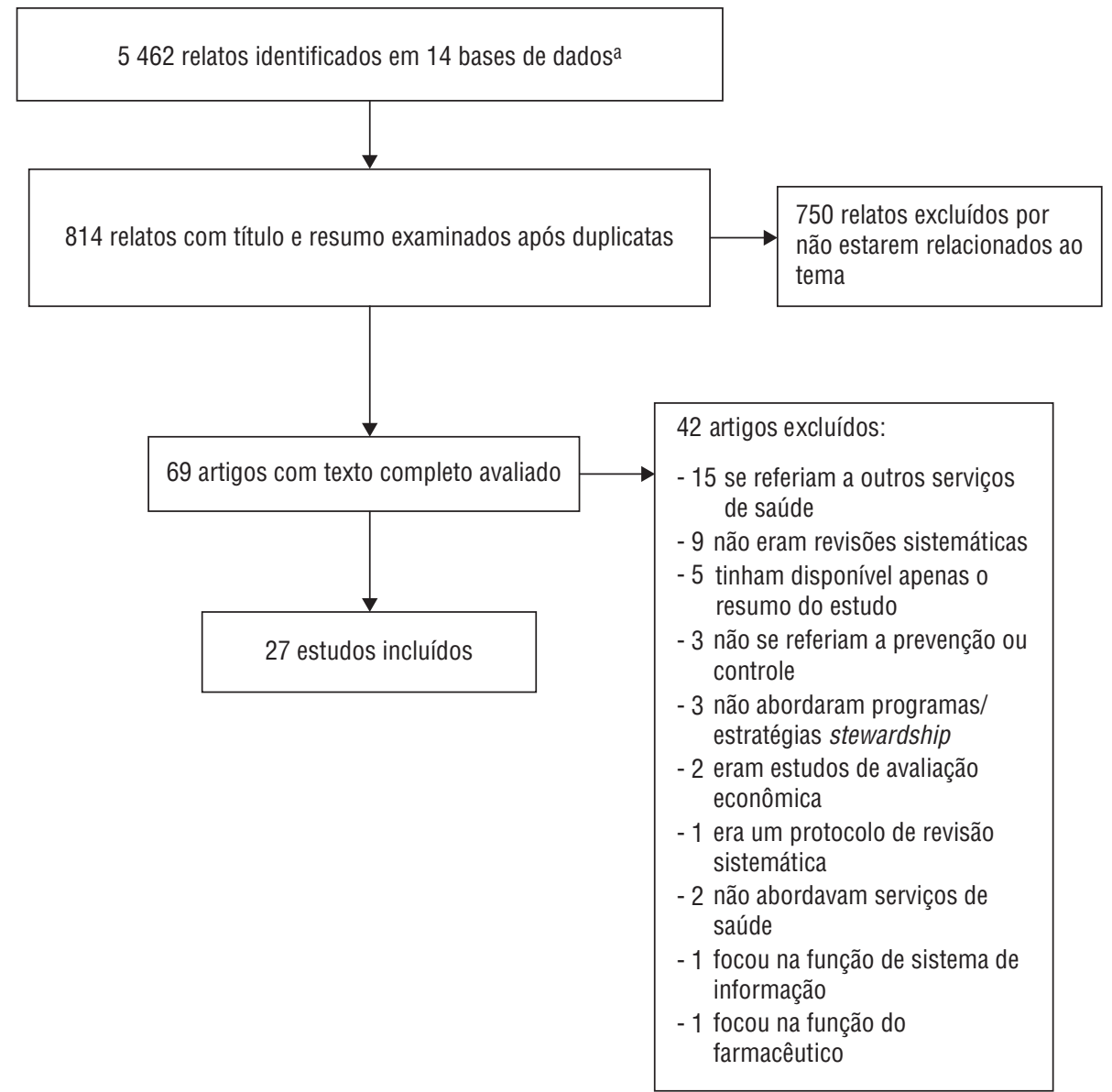

a Portal Regional da BVS (https://bvsalud.org/), MEDLINE via PubMed (https://www.ncbi.nlm.nih.gov/pubmed/), Health Systems Evidence (https://www.healthsystemsevidence.org/), PDQ Evidence (https://www.pdqevidence.org), Center for Reviews and Dissemination (https://www.crd.york.ac.uk/CRDWeb/), Embase (https://www.embase.com/login), Scopus (https://www.scopus.com/), Web of Science (http://www.webofscience. com/) , Cochrane Collaboration (https://www.cochranelibrary.com/), Proquest Dissertations and Theses Global (https://www.proquest.com/products-services/pqdtglobal.html), SisREBRATS (http://rebrats.saude.gov. br/sisrebrats), Biblioteca Digital Brasileira de Teses e Dissertações (http://bdtd.ibict.br/vufind/) e Joanna Briggs Institute (https://joannabriggs.org/ebp\#database). 
TABELA 1. Características das revisões sistemáticas incluídas na formulação das opções para políticas hospitalares de prevenção e controle de resistência aos antimicrobianos, listados por ordem decrescente de qualidade metodológica

Principais elementos das intervenções

Treinamento e restrição de uso de antimicrobianos (24)

Teste de procalcitonina (25)

Teste de procalcitonina (26)

- Aprovação prévia da prescrição por especialista em doenças infecciosas

- Intervenções persuasivas: educação, revisão e pós-prescrição e alterações nas diretrizes (27)

Rotação e restrição de antibióticos; restrição de um antibiótico e educação; restrição de antibióticos, auditoria e sistema de apoio à decisão; restrição de antibióticos e implementação de diretriz clínica; rotação de antibióticos; restrição de antibióticos e auditoria; higiene de mãos e administração de antibióticos (28)

Descalonamento de antimicrobianos (29)

Teste de procalcitonina (30)

Sistemas informatizados independentes de apoio à decisão; suporte à decisão incorporado ao prontuário médico eletrônico ou à entrada de pedidos de provedor informatizado; sistemas informatizados de aprovação antimicrobiana; sistemas de vigilância (31)

Restrição ou pré-aprovação de antibióticos, consulta médica formal sobre doenças infecciosas, implementação de protocolos de descalonamento, reavaliação formal de antibióticos em dia pré-especificado de terapia, diretrizes para profilaxia ou antibioticoterapia na UTI e implementação de suporte à decisão assistido por computador (13)

Pré-aprovação, auditoria e feedback prospectivos; educação; diretrizes; restrições de formulários; sistemas eletrônicos; e equipe multiprofissional (32)

Diretriz, manejo de antimicrobianos e ações educativas; algoritmo guiado por procalcitonina e dispositivo eletrônico (33)

Protocolos de procedimentos cirúrgicos, ações educativas (estudo de caso e guia de bolso, reuniões com especialistas, material didático), política de restrição a antibióticos e feedback (34)

Diretriz de monitoramento da terapia medicamentosa (35)

Suporte informatizado para tomada de decisão (36)

Teste de procalcitonina (37)

Diretrizes com feedback, formulário de restrição e pré-autorização (14)

Auditoria prospectiva e feedback, formulários de pré-autorização ou restrição, educação, orientação ou implementação de políticas, intervenção específica da síndrome, dose otimizada teste rápido de diagnóstico e sistema informatizado de apoio à decisão clínica (38)

Testes de proteína C reativa e interleucina-8 (39)

Materiais educacionais impressos / audiovisuais (formulários enviados, protocolos e diretrizes, materiais auto-instrucionais, boletins sobre fármacos); educação em grupo (sessões de grupo, conferências, palestras, seminários e tutoriais); feedback dos padrões de prescrição médica (individualmente ou comparação com o comportamento dos colegas e / ou padrões aceitos) ou feedback do paciente sobre listas específicas de medicamentos prescritos; visitas individuais de divulgação; lembretes durante a prescrição; sistemas de tomada de decisão auxiliados por computador; processo de controle de forma / forma restritiva; educação do paciente (panfletos, fitas de vídeo); workshops sobre testes rápidos / introdução de testes rápidos de antígenos em consultórios; aplicação de regulamentos; feedback sobre a prescrição com recomendações por farmacêuticos e / ou médicos de doenças infecciosas; incentivos financeiros (40)

\section{No. estudos}

Desfechos relatados ${ }^{b}$

AMSTAR

221

$\downarrow$ Duração da antibioticoterapia

$\downarrow$ Tempo de permanência hospitalar

$\downarrow$ Incidência de infecções por Clostridium difficile,

bactérias gram-negativas e gram-positivas resistentes

$26 \downarrow$ Duração da antibioticoterapia

$\downarrow$ Eventos adversos

$7 \downarrow$ Duração da antibioticoterapia

$16 \downarrow$ Incidência de infecções hospitalares

$76 \downarrow$ Incidência de infecções por bactérias Gram-negativas, Gram-negativas produtoras de beta-lactamase de espectro amplo,

Staphylococcus Aureus resistente à meticilina $\mathrm{e}$ Clostridium difficile

$20 \downarrow$ Taxa de mortalidade

$\uparrow$ Uso adequado de antimicrobianos

$\downarrow$ Quantidade de consumo de antibióticos

$\downarrow$ Eventos adversos

$45 \uparrow$ Uso adequado de antimicrobianos

10

8

8

8

$24 \downarrow$ Duração da antibioticoterapia

$\downarrow$ Quantidade de consumo de antibióticos

$\downarrow$ Eventos adversos

$\uparrow$ Uso adequado de antimicrobianos

$26 \downarrow$ Uso de antimicrobianos

$\downarrow$ Tempo de permanência hospitalar

$\downarrow$ Incidência de infecções hospitalares

$27 \downarrow$ Quantidade de consumo de antibióticos

$10 \downarrow$ Incidência de infecções hospitalares

$\uparrow$ Qualidade da prescrição

$145 \downarrow$ Taxa de mortalidade

$\uparrow$ Economia de recursos

$81 \downarrow$ Quantidade de consumo de antibióticos

$\downarrow$ Taxa de resistência antimicrobiana

$\uparrow$ Adequação da cobertura de antibióticos

$\uparrow$ Adesão às diretrizes de antibióticos

$16 \downarrow$ Duração da antibioticoterapia

$37 \downarrow$ Incidência de infecções por Clostridium difficile, bactérias gram-negativas e gram-positivas resistentes

$\downarrow$ Tempo de permanência hospitalar

$63 \downarrow$ Incidência de infecções por Clostridium difficile, bactérias gram-negativas e gram-positivas resistentes

$\downarrow$ Tempo de permanência hospitalar

$\downarrow$ Uso de antimicrobianos

$28 \downarrow$ Uso de antimicrobianos

$78 \downarrow$ Uso de antimicrobianos
6 
TABELA 1. Características das revisões sistemáticas incluídas na formulação das opções para políticas hospitalares de prevenção e controle de resistência aos antimicrobianos, listados por ordem decrescente de qualidade metodológica (continuação)

\begin{tabular}{|c|c|c|c|}
\hline Principais elementos das intervenções & No. estudos ${ }^{a}$ & Desfechos relatados ${ }^{b}$ & AMSTAR $^{c}$ \\
\hline $\begin{array}{l}\text { Auditoria prospectiva com feedback e utilização de formulários de restrição realizado pelo } \\
\text { farmacêutico clínico (15) }\end{array}$ & 28 & $\begin{array}{l}\downarrow \text { Incidência de infecções hospitalares } \\
\uparrow \text { Uso apropriado de antimicrobianos } \\
\downarrow \text { Taxa de mortalidade }\end{array}$ & 4 \\
\hline $\begin{array}{l}\text { Pré-autorização, auditoria prospectiva, feedback, revisão, consulta e recomendações, educação e } \\
\text { treinamento, diretrizes institucionais, sanções financeiras e relatórios públicos (10) }\end{array}$ & 77 & $\begin{array}{l}\downarrow \text { Taxa de mortalidade } \\
\downarrow \text { Tempo de permanência hospitalar } \\
\downarrow \text { Incidência de infecções hospitalares }\end{array}$ & 4 \\
\hline Diretriz, manejo de antimicrobianos e ações educativas (41) & 9 & $\downarrow$ Incidência de infecções hospitalares & 4 \\
\hline $\begin{array}{l}\text { Programa de stewardship; diretrizes } \\
\text { Inserção de farmacêutico na equipe (42) }\end{array}$ & 17 & $\uparrow$ Uso apropriado de antimicrobianos & 3 \\
\hline Cumprimento de guias de prática clínica (12) & 20 & $\uparrow$ Uso apropriado de antimicrobianos & 2 \\
\hline $\begin{array}{l}\text { Programas de stewardship pediátricos (auditoria prospectiva de } 72 \mathrm{~h} \text { com feedback, auditoria } \\
\text { prospectiva com feedback, diretrizes de restrição e prática clínica), administração de handshake } \\
\text { (cumprimento das ações por meio de incentivo do gestor) e estratégias de gerenciamento do } \\
\text { uso racional de antimicrobianos (equipes multidisciplinares, orientações práticas para sepse } \\
\text { tardia e enterocolite necrosante e auditoria prospectiva com feedback) (19) }\end{array}$ & (1) & $\begin{array}{l}\downarrow \text { Uso de antimicrobianos } \\
\downarrow \text { Gastos nas compras anuais dos antibióticos de } \\
\text { amplo espectro mais comuns }\end{array}$ & 1 \\
\hline $\begin{array}{l}\text { Ações de educação/orientação, formulário/restrição, revisão e feedback, assistência informática, } \\
\text { rotação de antimicrobiano (11) }\end{array}$ & 14 & $\begin{array}{l}\downarrow \text { Taxa de mortalidade } \\
\downarrow \text { Tempo de permanência hospitalar } \\
\downarrow \text { Duração da antibioticoterapia }\end{array}$ & 1 \\
\hline
\end{tabular}

\footnotetext{
Número de estudos primários incluídos.

l: redução; $\uparrow$ : aumento.
Qualidade metodológica alta: escore 8-11; moderada: 5-7; baixa: 0-4
}

das revisões sistemáticas. Foram identificadas diversas intervenções que têm sido utilizadas nos programa hospitalares de stewardship, de maneira combinada ou isoladamente: auditoria e feedback, emprego de biomarcadores, orientação por diretrizes clínicas, educação de profissionais e usuários, várias formas de manejo de antimicrobianos, equipe de gestão para otimizar o uso de antimicrobianos, handshake stewardship (gestão do uso de antimicrobianos por meio de auditoria e feedback realizadas pessoalmente pelo gestor do programa), higienização das mãos, processo de regulação dos prescritores e apoio de sistemas eletrônicos.

\section{Intervenções combinadas para gestão do uso racional de antimicrobianos}

As intervenções combinadas foram analisadas em 14 revisões sistemáticas, três de alta qualidade metodológica $(2,28$, $29)$, oito de qualidade moderada $(10,15,32-35,38,41)$, e três de baixa qualidade $(11,19,42)$. Evidenciou-se a eficácia de intervenções combinadas na redução do uso de antimicrobianos $(19,32,38)$, da mortalidade $(10,11,15,35)$, do tempo de permanência hospitalar $(10,11,32)$, da duração da antibioticoterapia $(11,33)$, da incidência de infecções hospitalares $(10,15,27,28$, $32,34)$ e da colonização bacteriana (28). Também foi relatada melhora na qualidade da prescrição (34) e no uso adequado de antimicrobianos $(15,42)$. Além disso, os programas mostraramse benéficos em contextos geriátricos (27) e ao longo do tempo (28).

Diferentes combinações de intervenções tornaram-se mais eficazes ao longo do tempo $(27,28)$. A implementação de programas de stewardship reduziu em $33,9 \%$ os custos de antimicrobianos (32) e em $62 \%$ os gastos com compras anuais dos antibióticos de amplo espectro mais comuns (19). Houve economia com o uso e adesão às diretrizes clínicas $(11,35)$, com o descalonamento da terapia habitual, com a transição de terapia intravenosa para oral e com o monitoramento da terapia medicamentosa (35). Nenhuma revisão sistemática trouxe relatos de eventos adversos relacionados às intervenções estudadas.

\section{Intervenção individual para gestão do uso racional de antimicrobianos}

Os programas de stewardship envolvendo uma única intervenção foram analisados em 16 revisões sistemáticas, das quais três analisaram tanto intervenções combinadas quanto únicas $(33,38,42)$. Seis revisões sistemáticas foram de alta qualidade $(24,29,31)$, oito de qualidade moderada $(13,14,33,36-40)$ e duas de baixa qualidade metodológica $(12,42)$.

A diminuição na duração da antibioticoterapia foi observada com a implementação de intervenções de stewardship de treinamento e restrição de uso de antimicrobianos (24), uso do teste de procalcitonina para apoio na tomada de decisão terapêutica $(25,26,37)$ e manejo de antimicrobianos, que pode ser realizado por meio de restrição ou pré-aprovação de antibióticos, consulta médica formal sobre doenças infecciosas, implementação de protocolos de descalonamento, reavaliação formal de antibióticos em dia pré-especificado de terapia, diretrizes para profilaxia ou antibioticoterapia em UTI e implementação de suporte à decisão assistido por computador (13). Houve diminuição do tempo de permanência hospitalar por funções de restrição e ativação (24), protocolos para conversão da terapia intravenosa em terapia oral (14), auditoria prospectiva e feedback, pré-autorização ou restrição de formulário, diretrizes clínicas ou implementação de políticas de uso de antibióticos, intervenção específica para síndromes, otimização de dose, testes de diagnóstico rápido e sistema informatizado de apoio à decisão clínica (38).

A redução de infecções por Clostridium difficile e por bactérias gram-negativas e gram-positivas resistentes foi observada com o uso de formulário de restrição e pré-autorização (14, 24), guias de prática clínica com feedback (14), auditoria prospectiva e feedback, pré-autorização ou restrição de formulário, 
diretrizes ou implementação de políticas, intervenção específica da síndrome, otimização de dose, testes de diagnóstico rápido e sistema informatizado de apoio à decisão clínica (14, 38). Constatou-se redução da mortalidade a partir do uso de sistema de suporte à tomada de decisão (29). O descalonamento associou-se a menos mortes nos casos de bacteriemia e sepse grave (29).

Houve redução do consumo de antibióticos com o uso de algoritmo guiado por procalcitonina $(30,33)$, guia de tratamento com antibióticos por meio de um dispositivo eletrônico (33), implementação de diretrizes com feedback, formulário de restrição e pré-autorização (14), manejo de antimicrobianos (13) e sistema de suporte à tomada de decisão (36). Destaca-se que não houve aumento de eventos adversos com o uso do teste de procalcitonina e o manejo de antimicrobianos $(13,30)$. O uso de procalcitonina, pelo contrário, mostrou-se associado a um menor risco de eventos adverso (25).

Uma redução no uso de antibióticos foi observada com a implementação de pré-autorização ou formulário de restrição, diretrizes ou implementação de políticas, intervenção específica da síndrome, otimização de dose, testes de diagnóstico rápido e sistema informatizado de apoio à decisão clínica (38). Resultados positivos no uso de antimicrobianos também foram identificados com programas educacionais (40) e com testes de proteína C reativa e interleucina-8 (39).

Observou-se que o uso adequado de antimicrobianos aumentou com a implementação de ações envolvendo tecnologias da informação (31), guia de tratamento com antibióticos por meio de um dispositivo eletrônico (30), formulário de restrição e pré-autorização, implementação de guias de prática sem feedback (14) e manejo de antimicrobianos (13). O uso apropriado de antimicrobianos em ambientes pediátricos foi alcançado com o cumprimento de recomendações de guias de prática clínica (12) e com a participação do farmacêutico clínico na equipe (42).

Uma redução na resistência antimicrobiana e melhora significativa da adequação da cobertura de antibióticos e maior adesão às diretrizes de antibióticos mostrou-se relacionada à implementação de sistema de suporte à decisão (36).

\section{Facilitadores e barreiras para a implementação da gestão do uso de antimicrobianos}

Os estudos indicam que são facilitadores da implementação de intervenções de stewardship em hospitais: satisfação de pacientes (36), ações educativas para profissionais de saúde e pacientes $(31,36)$, infraestrutura adequada (43), envolvimento dos profissionais de saúde na tomada de decisão e uma equipe multidisciplinar integrada $(10,12,14,33,44,45)$, relações de trabalho que propiciam um ambiente seguro para a prática clínica (15), integração de sistemas eletrônicos e qualidade dos serviços de laboratório $(45,46)$ e financiamento e planejamento com apoio da gestão $(44,45,47)$.

Os fatores que são barreiras para a implementação dos programas de gestão do uso de antimicrobianos relatados na literatura foram: falta de acesso a amostra de material de exames e insatisfação de pacientes $(36,48)$, diferenças culturais e de comportamento, falta de comunicação e de conhecimento empírico entre os profissionais $(12,24,26,34,40,41,45,47)$, defasagem na formação e treinamento dos profissionais inseridos nos serviços (45), dificuldades na organização do trabalho em decorrência da escassez e baixa fixação de profissionais ou acúmulo de funções, além de relações de trabalho que tornam o ambiente hostil aos profissionais de saúde $(44,47)$, falta de financiamento e de recursos humanos, infraestrutura inadequada e custo elevado das ações $(33,38)$.

\section{Contribuições do diálogo deliberativo}

As contribuições do diálogo deliberativo apresentadas neste artigo referem-se àquelas relacionadas a hospitais. Essas reflexões foram feitas de acordo com a realidade vivenciada pelos diversos participantes, que elencaram as principais barreiras e elementos facilitadores para implementação das estratégias de prevenção e controle de resistência a antimicrobianos. Cabe ressaltar que essas considerações, apresentadas a seguir, não compreendem um plano de ação ou de implementação.

Com relação aos usuários do sistema de saúde, um obstáculo à implementação dos programas de gestão do uso de antimicrobianos no hospital é a sua influência na prescrição - uma vez que os usuários esperam receber uma receita de antibiótico. Nesse sentido, há necessidade de educação comunitária, devendo-se abordar o problema da resistência antimicrobiana desde o ensino fundamental e de graduação. Aspectos de equidade das estratégias, de acordo com o contexto local, devem ser consideradas quando se trata de populações mais vulneráveis para garantir o cuidado em saúde sem discriminação.

No que se refere aos trabalhadores da saúde, foram apontadas como barreiras a falta de conhecimento técnico dos profissionais e gestores de saúde, a falta de conhecimento sobre diretrizes nacionais e a falta de recursos humanos, com sobrecarga de trabalho para os profissionais. Além disso, a ausência de equipe multidisciplinar, sobretudo a falta de farmacêuticos, é uma barreira que dificulta a implementação de programas de stewardship em hospitais.

As principais barreiras apontadas, contudo, foram as relacionadas à organização dos serviços hospitalares e sistemas de saúde, tais como ausência de fluxos de trabalho, falta de articulação entre os serviços, dificuldade de acesso a medicamentos de primeira linha e de alto custo no sistema público e no setor privado, falta de laboratórios e baixa qualidade e credibilidade dos laboratórios existentes e necessidade de sistemas eletrônicos que tenham interface com outros de serviços de saúde para facilitar a interlocução. Os conflitos de interesse, tanto do meio hospitalar (prescrição de medicamentos mais baratos) quanto da indústria farmacêutica, também podem contribuir para a prescrição inadequada de antimicrobianos.

Na esfera da macropolítica, foram mencionados a ineficiência das regulamentações vigentes, sobretudo no ambiente hospitalar, o caráter punitivo (em vez de educativo) da legislação, a necessidade de marcos regulatórios com base em evidências científicas que considerem especialmente a complexidade dos serviços e os indicadores de saúde, as mudanças políticas que afetam a condução das políticas públicas e o financiamento dessas políticas e o consumo elevado de antimicrobiano na indústria alimentícia e na agropecuária.

\section{DISCUSSÃO}

Os achados do presente artigo indicam que estratégias de stewardship combinadas ou individuais foram eficazes em diminuir o uso de antimicrobianos $(13,14,19,30,32,33,36,38-40)$, a 
mortalidade $(10,11,15,29,35)$, o tempo de permanência hospitalar $(10,11,14,24,32)$, a duração da antibioticoterapia $(11,13,24$, $26,33,34,26,37)$ e a incidência de infecções hospitalares $(10,14$, $24,27,28,32,34,38,41)$. Algumas revisões mostraram aumento no uso adequado de antimicrobianos (12-14, 30, 31, 42). Apenas uma revisão analisou como desfecho a redução de resistência microbiana e mostrou uma diminuição da taxa de resistência com suporte informatizado para tomada de decisão (36).

As revisões sistemáticas incluídas neste estudo mostraram a necessidade da realização de mais pesquisas de boa qualidade metodológica para explicar os problemas e desafios enfrentados pelos programas de stewardship $(15,38)$ e pelos programas que envolvem intervenções individuais $(13,33,35)$. Mesmo as revisões sistemáticas propriamente ditas carecem de maior rigor metodológico, uma vez que 21 delas apresentaram qualidade metodológica de moderada a baixa. Embora várias intervenções tenham se mostrado efetivas e seguras para prevenção e controle de resistência aos antimicrobianos em hospitais, sua implementação deve levar em consideração tais incertezas e lacunas do conhecimento. Ainda, sabe-se que, em geral, a implementação de intervenções combinadas tende a ser mais efetiva do que a implementação de intervenções individuais; no entanto, seria útil contar com mais estudos comparativos sobre a efetividade das intervenções únicas, uma vez que, em muitos locais, pode ser difícil implementar várias ações simultaneamente.

São limitações do presente estudo as estratégias de busca, que não incluíram termos como, por exemplo, aqueles relacionados à redução de infecções hospitalares. Embora as bases de dados pesquisadas tenham sido abrangentes, a ampliação nos termos de busca poderia contribuir para o levantamento de um número maior de estudos. A heterogeneidade de métodos utilizados e de intervenções incluídas nas revisões sistemáticas dificultou a análise da efetividade dessas ações $(27,32,35)$, gerando incertezas quanto ao real benefício de algumas intervenções $(27,32)$. De outro lado, observou-se uma escassez de pesquisas sobre programas de stewardship em serviços pediátricos (19), ou realizados em países de baixa e média renda $(32,41)$.

Em resumo, a implementação de programas para prevenção e controle da resistência a antimicrobianos em hospitais exige a elaboração de um plano de ação que considere o contexto local, as barreiras e os facilitadores. Esses programas exigem ainda a participação das partes interessadas na resolução do problema. Finalmente, é essencial a realização de estudos de alta qualidade metodológica que pesquisem os problemas e desafios associados aos programas de stewardship $(10,38)$.

Contribuição dos autores. Todas as autoras conceberam a ideia original. BCA e RCM coletaram e analisaram os dados. BCA e RCM interpretaram os resultados e escreveram o artigo. MCB e TST revisaram o artigo. Todas as autoras revisaram e aprovaram a versão final.

Agradecimentos. Às bibliotecárias da Biblioteca da Faculdade de Medicina da Universidade de Campinas pelo apoio no desenvolvimento da estratégia de busca e fornecimento dos artigos de acesso restrito.

\section{Conflitos de interesse. Nada declarado pelas autoras.}

Financiamento. Este artigo faz parte do projeto apoiado financeiramente pela chamada pública para projetos de Avaliação de Tecnologias em Saúde e de Políticas Informadas por Evidências sobre Resistência aos Antimicrobianos lançada em 2018 pelo Ministério da Saúde brasileiro (MS) e Organização PanAmericana da Saúde (OPAS) /Organização Mundial da Saúde (OMS).

Declaração. As opiniões expressas no manuscrito são de responsabilidade exclusiva dos autores e não refletem necessariamente a opinião ou política da $R P S P / P A J P H$ ou da Organização Pan-Americana da Saúde (OPAS).

\section{REFERÊNCIAS}

1. Ministério da Saúde. Secretaria de Ciência, Tecnologia e Insumos Estratégicos. Uso racional de medicamentos: temas selecionados / Ministério da Saúde, Secretaria de Ciência, Tecnologia e Insumos Estratégicos - Brasília: Ministério da Saúde, 2012 Disponível em: http://bvsms.saude.gov.br/bvs/publicacoes/uso_ racional_medicamentos_temas_selecionados.pdf. Acessado em 3 Setembro 2018.

2. Nathan C. Fresh Approaches to Anti-Infective Therapies. Sci Transl Med. 2012;4(140):140sr2. doi:10.1126/scitranslmed.3003081.

3. Word Health Organization. Antimicrobial resistance Global Report on Surveillance. Geneva: WHO; 2014. Disponível em: http:/ /apps. who.int/iris/bitstream/handle/10665/112642/9789241564748_ eng.pdf?sequence $=1$. Acessado em 9 Outubro 2019.

4. Word Health Organization. Antimicrobial Resistance: A Manual for developing national action plans. Geneva: WHO; 2017. Disponível em: http:/ /apps.who.int/iris/bitstream/handle/10665/204470/9789241 549530_eng.pdf?sequence=1. Acessado em 9 Outubro 2019.

5. Dallacorte TS, Indras DM, Teixeira JJV, et al. Prevalência e perfil de sensibilidade antimicrobiana de bactérias isoladas de hemoculturas realizadas em hospitais particulares. Rev Inst Adolfo Lutz. 2016;75:1702.

6. Waele JJ, Akova M, Antonelli M, Canton R, Carlet J, De Backer D, et al. Antimicrobial resistance and antibiotic stewardship programs in the ICU: insistence and persistence in the fight against resistance. A position statement from ESICM/ESCMID/WAAAR round table on multi-drug resistance. Intensive Care Med. 2018;44(2): 89-196.

7. ANVISA. Diretriz Nacional para Elaboração de Programa de Gerenciamento do Uso de Antimicrobianos em Serviços de Saúde. Brasília; 2017. Disponível em: http://portal.anvisa.gov.br/ documents /33852/271855/Diretriz+Nacional+para+Elabora $\%$ C3\%A7\%C3\%A3o+de+Programa+de+Gerenciamento+do+Uso+ de+Antimicrobianos+em+Servi $\%$ C3\%A7os+de+Sa\%C3\%BAde / 667979c2-7edc-411b-a7e0-49a6448880d4. Acessado em 9 Outubro 2019

8. Pan American Health Organization, Florida International University. Recommendations for Implementing Antimicrobial Stewardship Programs in Latin America and the Caribbean: Manual for Public Health Decision-Makers. Washington, D.C.: PAHO, FIU; 2018 Disponível em: https://iris.paho.org/handle/10665.2/49645. Acessado em 10 Marco 2020.

9. Word Health Organization. Global antimicrobial resistance surveillance system (GLASS) report: early implementation 20162017. Geneva: WHO; 2017. Disponível em: http://apps.who.int/ iris/ bitstream/handle/10665/259744/9789241513449-eng.pdf? sequence=1. Acessado em 9 Outubro 2019. 
10. Lee CF, Cowling BJ, Feng S, Aso H, Wu P, Fukuda K et al. Impact of antibiotic stewardship programmes in Asia: a systematic review and meta-analysis. J Antimicrob Chemother. 2018;73(4):844-851.

11. Bosso JA, Drew RH. Application of antimicrobial stewardship to optimise management of community acquired pneumonia. Int $\mathrm{J}$ Clin Pract. 2011; 65(7):775-83.

12. Nasr Z, Paravattil B, Wilby KJ. The impact of antimicrobial stewardship strategies on antibiotic appropriateness and prescribing behaviours in selected countries in the Middle East: a systematic review. East Mediterr Health J. 2017;23(6):430-440.

13. Kaki R, Elligsen M, Walker S, Simor A, Palmay L, et al. Impact of antimicrobial stewardship in critical care: a systematic review. J Antimicrob Chemother. 2011;66(6):1223-1230. doi:10.1093/jac/ dkr137j.

14. Wagner B, Filice G, Drekonja D, Greer N, MacDonald R, Rutks I. et al. Antimicrobial stewardship programs in inpatient hospital settings: a systematic review. Infect Control Hosp Epidemiol. 2014;35(10):1209-28

15. Mas-Morey P, Valle M A systematic review of inpatient antimicrobial stewardship programmes involving clinical pharmacists in smallto-medium-sized hospitals. Eur J Hosp Pharm. 2018;25(e1):e69-e73. doi:10.1136/ejhpharm-2017-001381.

16. Dyar OJ, Huttner B, Schouten J, et al. What is antimicrobial stewardship? Clin Microbiol Infect. 2017;23(11):793-8.

17. ANVISA. Plano de Ação da Vigilância Sanitária em Resistência aos Antimicrobianos. Brasília; 2018. Disponível em: http:// portal.anvisa.gov.br/documents / 3487091/3697444/Plano+ de+a $\%$ C3 $\%$ A $7 \%$ C3 $\%$ A3o+da+vigil $\%$ C $3 \%$ A2ncia + sanit $\%$ C $3 \%$ A1ria/09f85d62-bc23-4ccf-8c86-0a6431a355f9. Acessado em 9 Outubro 2019.

18. ANVISA. Plano Nacional para a Prevenção e o Controle da Resistência Microbiana nos Serviços de Saúde. Brasília; 2017. Disponível em: http:/ / portal.anvisa.gov.br/documents/33852/3074203/ Boletim+de+Seguran $\%$ C3\%A7a + do + Paciente+e+Qualidade+em + Servi $\%$ C3\%A7os+de+Sa $\%$ C3 $\%$ BAde+n $\%$ C2\%BA $+14+$ Avalia $\%$ $\mathrm{C} 3 \% \mathrm{~A} 7 \% \mathrm{C} 3 \% \mathrm{~A} 3 \mathrm{o}+$ dos+indicadores+nacionais+das+Infec $\% \mathrm{C} 3 \% \mathrm{~A} 7 \%$ C3\%B5es+Relacionadas+ $\%$ C $3 \%$ A $0+$ Assist $\%$ C $3 \%$ AAncia $\%$ C3\%A0+Sa $\%$ C3\%BAde+\%28IRAS\%29+e+Resist $\%$ C3\%AAncia+ microbiana/dbd57c96-937f-45d3-93fd-e76684b7f35c. Acessado em 9 Outubro 2019.

19. Godbout EJ, Pakyz AL, Markley JD, Noda AJ, Stevens MP. Pediatric Antimicrobial Stewardship: State of the Art. Curr Infect Dis Rep. 2018;20(10):39.

20. Lavis JN, Oxman AD, Lewin S, Fretheim A. Ferramentas SUPPORT para a elaboração de políticas de saúde baseadas em evidências (STP); 2009. Disponível em: http://sintese.evipnet. net/livro/ferramentas-support-para-a-elaboracao-de-politicasde-saude-baseadas-em-evidencias-stp/. Acessado em 18 Fevereiro 2019.

21. Toma TS, Tesser TR, Setti C, Bortoli MC. Síntese de evidências para políticas de saúde. Em: Toma et al (Org). Avaliação de tecnologias de saúde \& políticas informadas por evidências. São Paulo: Instituto de Saúde; 2017. Disponível em: http://www.saude.sp. gov.br/resources/instituto-de-saude/homepage/pdfs/avaliacao_ tecnologia_saudepolticas_inf_evidencias.pdf. Acessado em 9 Outubro 2019.

22. Shea BJ, Grimshaw JM, Wells GA, Boers M, Andersson N, Hamel $\mathrm{C}$ et al. Development of AMSTAR: a measurement tool to assess the methodological quality of systematic reviews. BMC Med Res Methodol. 2007;7:10. doi:10.1186/1471-2288-7-10.

23. Lavis JN, Boyko J, Oxman AD, Lewin S, Fretheim A. SUPPORT Tools for evidence-informed health Policymaking (STP). 14. Organising and using policy dialogues to support evidence-informed policymaking. Health Res Policy Syst. 2009;7(Suppl 1):S14. doi:10.1186/1478-4505-7-S1-S14.

24. Davey P, Marwick CA, Scott CL, Charani E, McNeil K et al. Interventions to improve antibiotic prescribing practices for hospital inpatients. Cochrane Database Syst Rev. 2017;2(2):CD003543. doi:10.1002/14651858.CD003543.pub4.

25. Schuetz P, Wirz Y, Sager R, Christ-Crain M, Stolz D, Tamm $M$, et al. Procalcitonin to initiate or discontinue antibiotics in acute respiratory tract infections. Cochrane Database Syst Rev. 2017;10(10):CD007498. doi:10.1002/14651858.CD007498.pub3.
26. Kopterides P, Siempos II, Tsangaris I, Tsantes A, Armaganidis A. Procalcitonin-guided algorithms of antibiotic therapy in the intensive care unit: a systematic review and meta-analysis of randomized controlled trials. Crit Care Med. 2010;38(11):2229-41.

27. Feazel LM, Malhotra A, Perencevich EN, Kaboli P, Diekema DJ, Schweizer ML. Effect of antibiotic stewardship programmes on Clostridium difficile incidence: a systematic review and metaanalysis. J Antimicrob Chemother. 2014;69(7):1748-54.

28. Baur D, Gladstone BP, Burket F, Carrara E, Foschi F, Döbele S, et al. Effect of antibiotic stewardship on the incidence of infection and colonisation with antibiotic-resistant bacteria and Clostridium difficile infection: a systematic review and meta-analysis. Lancet Infect Dis. 2017;17:990-1001.

29. Paul M, Dickstein Y, Raz-Pasteur A. Antibiotic de-escalation for bloodstream infections and pneumonia: systematic review and meta-analysis. Clin Microbiol Infect. 2016;22(12):960-7

30. van der Does Y, Rood PP, Haagsma JA, Patka P, van Gorp EC, Limper M. Procalcitonin-guided therapy for the initiation of antibiotics in the ED: a systematic review. Am J Emerg Med. 2016;34(7): 1286-93.

31. Baysari MT, Lehnbom EC, Li L, Hargreaves A, Day RO, Westbrook JI. The effectiveness of information technology to improve antimicrobial prescribing in hospitals: A systematic review and meta-analysis. Int J Med Inform. 2016;92:15-34.

32. Karanika S, Paudel S, Grigoras C, Kalbasi A, Mylonakis E. Systematic Review and Meta-analysis of Clinical and Economic Outcomes from the Implementation of Hospital-Based Antimicrobial Stewardship Programs. Antimicrob Agents Chemother. 2016;60(8):4840-52. doi:10.1128/AAC.00825-16.

33. Van Dijck C, Vlieghe E, Cox JA. Antibiotic stewardship interventions in hospitals in low-and middle-income countries: a systematic review. Bull World Health Organ. 2018;96(4):266-80. doi:10.2471/ BLT.17.203448.

34. Charani E, Edwards R, Sevdalis N, Alexandrou B, Sibley E, Mullett $\mathrm{D}$ et al. Behavior change strategies to influence antimicrobial prescribing in acute care: a systematic review. Clin Infect Dis. 2011;53(7):651-62.

35. Schuts EC, Hulscher MEJL, Mouton JW, Verduin CM, Stuart JWTC, Overdiek HWPM et al. Current evidence on hospital antimicrobial stewardship objectives: a systematic review and meta-analysis. Lancet Infect Dis. 2016;16(7):847-56.

36. Curtis CE, Al Bahar F, Marriott JF. The effectiveness of computerised decision support on antibiotic use in hospitals: A systematic review. PLoS One. 2017;12(8):e0183062. doi:10.1371/journal.pone. 0183062.

37. Shafiq N, Gautam V, Pandey AK, Kaur N, Garg S, Negi H, et al. A meta-analysis to assess usefulness of procalcitonin-guided antibiotic usage for decision making. Indian J Med Res. 2017;146(5): $576-84$.

38. Honda H, Ohmagari N, Tokuda Y, Mattar C, Warren DK. Antimicrobial Stewardship in Inpatient Settings in the Asia Pacific Region: A Systematic Review and Meta-analysis. Clin Infect Dis. 2017;15;64(suppl_2):S119-S126.

39. Patel SJ, Larson EL, Kubin CJ, Saiman L. A review of antimicrobial control strategies in hospitalized and ambulatory pediatric populations. Pediatr Infect Dis J. 2007;26(6):531-7.

40. Roque F, Herdeiro MT, Soares S, Teixeira Rodrigues A, Breitenfeld L, Figueiras A. Educational interventions to improve prescription and dispensing of antibiotics: a systematic review. BMC Public Health. 2014;14:1276. doi:10.1186/1471-2458-14-1276.

41. Silva ARA, Dias DCA, Marques AF, Biase CB, Murni IK, Dramowski A, et al. Role of antimicrobial stewardship programmes in children: a systematic review. J Hosp Infect. 2018;99(2):117-23.

42. Smith MJ, Gerber JS, Hersh AL. Inpatient Antimicrobial Stewardship in Pediatrics: A Systematic Review. J Pediatric Infect Dis Soc. 2015;4(4):e127-35.

43. Barbut F, Jones G, Eckert C. Epidemiology and control of Clostridium difficile infections in healthcare settings: an update. Curr Opin Infect Dis. 2011;24(4):370-6.

44. Bal AM, Gould IM. Antibiotic stewardship: overcoming implementation barriers. Curr Opin Infect Dis. 2011;24(4):357-62.

45. Blumenthal KG, Shenoy ES, Wolfson AR, Berkowitz DN, Carballo VA, Balekian DS, et al. Addressing Inpatient Beta-Lactam Allergies: 
A Multihospital Implementation. J Allergy Clin Immunol Pract. 2017;5(3):616-25. doi:10.1016/j.jaip.2017.02.019.

46. Cox JA, Vlieghe E, Mendelson M, et al. Antibiotic stewardship in low- and middle-income countries: the same but different? Clin Microbiol Infect. 2017;23(11):812-8. doi:10.1016/j.cmi.2017.07.010n.

47. van Buul LW, Sikkens JJ, van Agtmael MA, et al. Participatory action research in antimicrobial stewardship: a novel approach to improving antimicrobial prescribing in hospitals and long-term care facilities. J Antimicrob Chemother . 2014;69(7):1734-41.

48. Derde LP, Dautzenberg MJ, Bonten MJ. Chlorhexidine body washing to control antimicrobial-resistant bacteria in intensive care units: a systematic review. Intensive Care Med. 2012;38(6):931-9. doi:10.1007/s00134-012-2542-z.

Submetido em 20 de novembro de 2019. Aceito em versão revisada em 4 de fevereiro de 2020.

\section{Prevention and control of antimicrobial stewardship: a review of evidence}

ABSTRACT Objective. To identify effective interventions to manage antimicrobial resistance in hospital settings and potential barriers to their implementation.

Method. A synthesis of evidence for health policy was performed using SUPPORT tools. Literature searches were performed in November and December 2018 in 14 databases. A face-to-face deliberative dialogue workshop to identify implementation barriers was performed with 23 participants (manager, researchers, and health care professionals) and 14 listeners divided into three groups. Researchers with experience in deliberative dialogue acted as facilitators.

Results. Twenty-seven systematic reviews focusing on antimicrobial stewardship using combined or individual strategies were identified. The interventions included education, electronic systems, use of biomarkers, and several strategies of antimicrobial management. The main barriers to the implementation of interventions, identified in the literature and deliberative dialogue workshop, were poor infrastructure and insufficient human resources, patient complaints regarding the treatment received, cultural differences within the multidisciplinary team, work overload, and lack of financing/planning.

Conclusion. Most of the strategies identified were effective for antimicrobial stewardship in hospital settings. The reliability of results may be strengthened with the performance of additional research of higher methodological quality.

Keywords Drug resistance, microbial; antimicrobial stewardship; hospitals; evidence-informed policy. 


\section{Gestión de las intervenciones en materia de prevención y control de la resistencia a los antimicrobianos en los hospitales: revisión de la evidencia}

RESUMEN Objetivo. Determinar cuáles son las intervenciones eficaces para enfrentar la resistencia a los antimicrobianos en los hospitales y los posibles obstáculos para su implementación.

Métodos. Se hizo una síntesis de la evidencia encontrada para la elaboración de políticas con base en la metodología propuesta en las herramientas SUPPORT. Se efectuaron búsquedas bibliográficas en 14 bases de datos en noviembre y diciembre del 2018. Con el fin de determinar los obstáculos para la implementación de las intervenciones, se llevó a cabo un diálogo deliberativo en modalidad presencial con 23 participantes (gerentes, investigadores y profesionales de salud) y 14 oyentes, divididos en tres grupos. Varios investigadores con experiencia en la realización de diálogos deliberativos integraron el grupo de moderadores.

Resultados. Se encontraron 27 revisiones sistemáticas conjuntas e individuales de intervenciones para la gestión de los antimicrobianos (programas de rectoría). En esas intervenciones se abordaron estrategias de educación, sistemas electrónicos, biomarcadores y diversas formas de manejo de los antimicrobianos. Los principales obstáculos para la implementación de las intervenciones, detectadas por medio de la literatura y del diálogo deliberativo, fueron la falta de infraestructura y de recursos humanos, la insatisfacción del paciente con el comportamiento terapéutico, las diferencias culturales y la sobrecarga de trabajo del equipo multidisciplinario, así como la falta de financiamiento y planificación.

Conclusión. En su mayoría, las estrategias encontradas demostraron ser eficaces para la gestión de la resistencia a los antimicrobianos en el ámbito hospitalario. Cabe destacar que con nuevas investigaciones de mejor calidad metodológica podría aumentarse la confianza en los resultados.

Palabras clave Farmacorresistencia microbiana; programa de optimización del uso de los antimicrobianos; hospitales; política informada por la evidencia. 